\title{
The speciality of Emergency Medicine - a global perspective
}

\author{
Cameron $\mathrm{PA}^{1}$ \\ Journal of the Ceylon College of Physicians, 2017, 48, 8-10
}

\section{Background}

Care for the acutely ill and injured has changed considerably over the last few decades. Technological and scientific discoveries have allowed major breakthroughs in the way we manage patients. Although available emergency treatments have been similar across continents, there have historically been large differences in the systems of care and the training of clinicians. In the Anglo-American sphere of influence, there was a rapid development of the specialty of emergency medicine and emergency medical systems from the late 1960s through to the $2000 \mathrm{~s}^{1,2}$. In other parts of the world, emergency medicine as a specialty took longer to develop and emergencies were managed under traditional sub-specialties according to presumed aetiology or by junior "undifferentiated" doctors ${ }^{3}$.

The development of the specialty of emergency medicine has been driven by a number of factors. The most important driver has been the fact that we can now actually improve patient outcomes by applying evidence based clinical pathways to a large number of conditions - something that was not possible 100 years ago. Emergency conditions such as cardiac arrest, AMI, stroke, trauma and sepsis, have been shown to have improved outcomes with the right treatment given early by appropriately trained clinicians $s^{4,5,6,7,8}$. Other factors have also influenced changes to emergency care. With the explosion in medical knowledge, it is not possible for any one generalist physician to know all about any subject. Subspecialisation has allowed doctors to know a lot about a narrow breadth of medical care (vertical specialisation). However when a complex patient with

\footnotetext{
${ }^{1}$ National Trauma Research Institute, The Alfred Hospital, Melbourne, Australia;
}

Emergency and Trauma Centre, The Alfred Hospital, Melbourne, Australia;

Monash University, Melbourne, Australia;

Past President, International Federation of Emergency Medicine (IFEM).

E-mail: peter.cameron@monash-edu multiple illnesses/complications is being managed by a single subspecialty doctor, this can be hazardous. A patient with diabetes, might have renal failure, heart failure, peripheral neuropathy and blindness - a fall in this patient may be precipitated by multiple causes. Which specialty can best assess a complicated diabetic patient with an injury following a fall? With ageing of the population and survival of patients with chronic illnesses (eg. cystic fibrosis, oncology, transplants), single system illnesses are becoming less common.

Improved transport systems allow patients to travel large distances over short timeframes, either by road or air. Thus, patient management can be regionalised and prehospital triage by ambulance allows patients to be transported directly to major centres when appropriate. Technological advances also allow transfer of images and "tele-health" consultations across regions.

Historically, a century ago, most medicine was delivered in the home and the community. With technological advances, it was no longer possible to deliver complicated treatments outside of institutions. For example Xray machines, CT scans, laboratories, monitoring, specialist teams and operating theatres were only practical at an institutional level. This led to the rise of hospital medicine. Because of hospital medicine, there was a need for a method of sorting and treating patients at "the Front door" - the emergency department. Ironically, just as emergency medicine is becoming established, increasing miniaturisation and improvements in data transfer, the ability to monitor, image, assess and treat in the community may change the flow of patients to institutions (hospitals) and back into the community. However, there will still need to be generalists who coordinate specialist emergency care, even if this is no longer practised at a physical hospital facility.

With hospital medicine and institutional care, came the issue of how to process patients and how to develop systems of care. This coincided with the factory process engineering developments of last century associated with manufacturing. Industrial processes were designed to ensure efficiency and improve quality. The theoretical constructs for this came from industry rather than medicine. Emergency medicine and the emergency department became the first step in the conveyer belt of hospital emergency care. Medicine became another

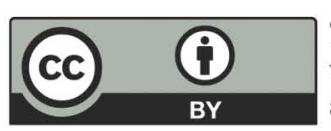

This is an open-access article distributed under the terms of the Creative Commons Attribution License, which permits unrestricted use, distribution, and reproduction in any medium, provided the original author and source are credited. 
industrial process - emergency medicine was necessary at the commencement of hospital management of illnesses to ensure consistency of processes. Models of care to improve efficiency and safety such as triage ${ }^{9}$, short stay medicine (10) and facilitated discharge ${ }^{11}$ were part of this evolution.

Patients, the community and politicians have increasingly expected more from the medical system. Increased knowledge through education, the internet and "Dr Google" have changed the nature of the doctorpatient relationship. The paternalistic approach from decades ago is no longer accepted. Unless doctors can follow the best evidence and ensure access to the latest management, they are open to litigation and even anger from patients and relatives manifest in violence. The specialty of emergency medicine and an emergency medical system, allow hospitals to have con-sistent, evidence based processes for all emergency presentations.

Emergency medicine is defined by the International Federation for Emergency Medicine (IFEM) as "a field of practice based on the knowledge and skills required for the prevention, diagnosis and management of acute and urgent aspects of illness and injury affecting patients of all age groups with a full spectrum of episodic undifferentiated physical and behavioural disorders; it further encompasses an understanding of the deve-lopment of prehospital and inhospital emergency medical systems and the skills necessary for this development"12.

It is a broad based specialty, which covers a small part of many "vertical" specialties. That is, it is the "horizontal" base that provides the core knowledge and skills $24 / 7$ for all the subspecialties of medical care that have developed. There are some components which are specific to emergency medicine and not necessarily covered by traditional specialties, such as prehospital care, disaster medicine and toxicology.

\section{Training}

The generic training requirements for emergency medicine have been agreed by specialist societies globally through the IFEM ${ }^{13,14}$. Despite general agreement regarding the skills and clinical exposure necessary to become a good emergency physician, the length of training programs globally varies from 3-7 years and mandated clinical rotations also vary. There are also options for subspecialist fellowship training programs, such as critical care, hyperbaric and academic streams in some countries. The approach to training is to some extent determined by funding, payment models and historical training structures for other specialties.

\section{CME}

The broad based clinical skills necessary to provide excellent emergency care require ongoing training, throughout the professional career of an emergency specialist. There are different approaches in different countries. Some countries mandate recertification, others insist on documentation of regular updates and skills training ${ }^{15}$. There is no evidence that one approach is better than another.

\section{Funding}

The funding of emergency care varies around the world. Some governments use the "fire brigade" principle - EM is a crucial part of the infrastructure and is paid for from general revenue. Access to emergency care is free, with perhaps a nominal payment. Other jurisdictions use a fee for service model, or casemix payment with each visit and procedure paid for by the patient or insurer ${ }^{16}$. The method of payment is to some extent determined by how doctors and hospitals are paid for generally - and thus it is not possible to state that one form of funding is better than another. Whichever method is used to fund emergency care, there has to be some legislated safety nets to ensure all critically ill patients receive basic emergency care.

\section{Is trauma a separate specialty?}

Emergency and trauma clinical work often overlaps and the initial reception and resuscitation is frequently undertaken by emergency physicians. Surgeons have historically seen trauma care as a surgical disease, however, increasingly, with the preponderance of blunt trauma and an ageing population, the vast majority of patients have no surgery other than orthopaedics. The skills of emergency physicians, enabling accurate assessment, quick decision making, coordination and resuscitation, are needed in all trauma patients. Globally, many emergency physicians are not only taking a role as team leader in the initial assessment of trauma patients, but also in the ongoing coordination of care beyond the ED. Importantly, trauma care involves a team of clinicians with multidisciplinary skills and coordination of care from roadside through to rehabilitation. Emergency medicine has a fundamental role in ensuring the initial reception and resuscitation are optimal.

\section{Disaster preparedness}

Recent events in many parts of the world have demonstrated the importance of a robust emergency system, in the preparation for an external disaster. Disasters, by definition, are difficult to predict. They also take many forms - earthquakes, floods, infectious disease and terrorist threats for example. A well coordinated emergency system, with trained emergency physicians to lead the institutional response will inevitably lead to better outcomes. 


\section{The future}

It seems inevitable that emergency medicine will continue to evolve and prosper as the delivery of medical care becomes more complex. The need to have experts with assessment and resuscitation skills available 24/7 is unarguable for a safe and effective emergency system $^{17,18,19}$. The only means of providing this, is by a targeted specialist training program. Sri Lanka is now part of a global movement to improve access to emergency care - a basic human right.

\section{References}

1. Australasian College for Emergency Medicine. History of ACEM.[Cited 2017.] Available from URL: http:// www.acem.org.au

2. Suter R. Emergency medicine in the United States: a systemic review. World J. Emerg. Med. 2012; 3: 5-10.

3. Dick WF. American vs. Franco-German emergency medical services system. Prehospital Disaster Med. 2003; 18: 29-37.

4. Beck CS, Pritchard WH, Feil HS. Ventricular fibrillation of long duration abolished by electric shock. JAMA 1947; 135: 985.

5. Fleming A. 'On the antibacterial action of cultures of a penicillium, with special reference to their use in the isolation of B. influenzae'. Br. J. Exp. Pathol. 1929; 10: 226-36.

6. Stub D, Bernard S, Smith K, et al. Do we need cardiac arrestcentres in Australia? Int. Med. J. 2012; 42: 1173-9.

7. Cameron PA, Gabbe BJ, Cooper JD, et al. A statewide system of trauma care in Victoria: effect on patient survival. Med. J. Aust. 2008; 189: 546-50.

8. The National Institute of Neurological Disorders and Stroke rt-PA Stroke Study Group. Tissue plasminogen activator for acute ischemic stroke. N Engl J Med. 1995; 333: 1581-7.
9. Oredsson $\mathrm{S}$, Jonsson $\mathrm{H}$, Rogne $\mathrm{J}$, et al. A systematic review of triage-related interventions to improve patient flow in emergency departments. Scand. J. Trauma Resusc. Emerg. Med. 2011; 19: 43.

10. Daly S, Campbell DA, Cameron PA. Short-stay units and observation medicine: a systematic review. Med. J. Aust. 2003; 178: 559-63.

11. Moss JE, Houghton LM, Flower CL, Moss DL, Nielsen DA,McD Taylor D. A multidisciplinary Care Coordination Team improves emergency department discharge planning practice. Med. J. Aust. 2002; 177: 435-9.

12. International Federation for Emergency Medicine IFEM. Definition of Emergency Medicine. [Cited 2017] Available fromURL: http://www.ifem.cc

13. Hobgood C, Anantharaman V, Bandiera G, et al. InternationalFederation for Emergency Medicine model curriculum for medical student education in emergency medicine. Emerg. Med. Australas. 2009; 21: 367-72.

14. Hobgood C, Anantharaman V, Bandiera G, et al. International Federation for Emergency Medicine Model Curriculum for Emergency Medicine Specialists. Emerg. Med. Australas. 2011; 23: 541-53.

15. Hobgood C, Mulligan T, Bodiwala G, Cameron P, Holliman, J. (Jim), Kwan, J, Singer A, Jouriles N. 'International Federation for Emergency Medicine Model Curriculum for Continuing Professional Development' CJEM 2015; 17(3): 295-309. doi: 10.1017/cem.2014.79

16. Duckett SJ, Jackson T. Paying for hospital care under a single payer system. Ann. Emerg. Med. 2001; 37: 309-17.

17. Holliman CJ, Mulligan TM, Suter RE, et al. The efficacy and value of emergency medicine: a supportive literature review. Int. J. Emerg. Med. 2011; 4: 44.

18. Razzak J, Kellerman A. Emergency medical care in developing countries; is it worthwhile? Bull. World Health Org. 2002; 80: 900-5.

19. Cameron P. Are we the systems specialists? Emerg. Med. (Fremantle) 2003; 15: 1-3. 